\title{
Graphene Nanoribbon Conductance Model in Parabolic Band Structure
}

\author{
Mohammad Taghi Ahmadi, Zaharah Johari, N. Aziziah Amin, \\ Amir Hossein Fallahpour, and Razali Ismail
} Department of Electronic Engineering, Faculty of Electrical Engineering, Universiti Teknologi Malaysia, Skudai 81310,
Johor Darul Takzim, Malaysia

Correspondence should be addressed to Mohammad Taghi Ahmadi, ahmadiph@gmail.com

Received 15 April 2010; Accepted 15 June 2010

Academic Editor: Rakesh K. Joshi

Copyright (C) 2010 Mohammad Taghi Ahmadi et al. This is an open access article distributed under the Creative Commons Attribution License, which permits unrestricted use, distribution, and reproduction in any medium, provided the original work is properly cited.

\begin{abstract}
Many experimental measurements have been done on GNR conductance. In this paper, analytical model of GNR conductance is presented. Moreover, comparison with published data which illustrates good agreement between them is studied. Conductance of GNR as a one-dimensional device channel with parabolic band structures near the charge neutrality point is improved. Based on quantum confinement effect, the conductance of GNR in parabolic part of the band structure, also the temperature-dependent conductance which displays minimum conductance near the charge neutrality point are calculated. Graphene nanoribbon (GNR) with parabolic band structure near the minimum band energy terminates Fermi-Dirac integral base method on band structure study. While band structure is parabola, semiconducting GNRs conductance is a function of Fermi-Dirac integral which is based on Maxwell approximation in nondegenerate limit especially for a long channel.
\end{abstract}

\section{Introduction}

Graphene consist of a single sheet of cabon atom bonded in $\mathrm{sp} 2$ of hexagonal lattice structure offers a numbers of fascinating possibilities in electronics application $[1,2]$. Graphene is a gapless two dimensional material which its confinement introduce one dimensional Graphene nanoribbon with a width less than the De-Broglie wavelength as illustrated in Figure 1. The formation of band gap in GNR resulted from the confinement of electron that form standing waves along the chiral vector [3,4]. The band gap of the GNR formed that depends on its width and chirality leads to different carrier transport phenomena [5, 6]. GNR band structure shows electronic properties of metallic and semiconducting just like the CNT [7]. GNR also share similar properties with single wall CNTs (SWNTs) [8] that have a mean free path in a range of micrometer $[9,10]$ as well as carrying higher carrier densities. Unlike CNTs, GNRs has simpler fabrication process owing to excellent future electronic devices like transistor and interconnect $[11,12]$. Theoretical study on GNR [13-15] is still at the beginning stage and the conductance phenomena based on band structure are still unexplored. In this paper, physical model of GNR conductance presented as a function of normalized Fermi energy.

\section{Conductance Modeling}

Applying the Taylor expansion on graphene band energy near the Fermi point, the $E(k)$ relation of the GNR is obtained as [16]

$$
E(\vec{k})= \pm \frac{t 3 a_{C-C}}{2} \sqrt{k_{x}^{2}+\beta^{2}}
$$

where $\beta$ is quantized wave vector given by [17]

$$
\beta=\frac{2 \pi}{a_{c-c} \sqrt{3}}\left(\frac{p_{i}}{N+1}-\frac{2}{3}\right)
$$

here $p_{i}$ is the subband index and $N$ is the number of dimmer lines which determine the width of the ribbon [17] and $k_{x}$ 


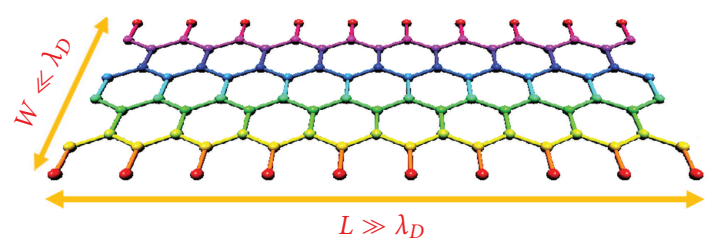

Figure 1: A prototype one-dimensional GNR with $W \ll \lambda_{D}$ and $L \gg \lambda_{D}$ for rectangular cross-section.

is the wave vector along the length of the nanoribbon. The energy band gap can be assumed as

$$
E_{g}=3 t a_{c c} \beta \text {. }
$$

Therefore, the modified energy relation is

$$
E= \pm \frac{E_{g}}{2} \sqrt{\left(1+\frac{k_{x}^{2}}{\beta^{2}}\right)}
$$

Equation (5) which indicates relationship between energy and wave vector is not parabolic, however, Square root approximation in corporate with Taylor expansion leads to parabolic relation between energy and wave vector $[18,19]$

$$
\begin{gathered}
E(k) \approx \frac{E_{g}}{2}+\frac{E_{g}}{4 \beta^{2}} k_{x}^{2}, \\
E \approx \frac{E_{g}}{2}+\frac{\hbar^{2} k_{x}^{2}}{2 m^{*}},
\end{gathered}
$$

where $m^{*}$ is the effective mass of GNR. Plotting the GNR energy band of (5) and (7) in Figure 2, it is obviously shown that the band structure is parabolic at certain range of energy in $E-k$ relationship. In parabolic part of the band energy, the wave vector can be extracted as

$$
k=\sqrt{\frac{4 E}{3 a_{c c} t}-2 \beta^{2}} .
$$

Based on this wave vector, number of actual modes $M(E)$ at a given energy which is dependent on the subbands location can be calculated. If the related energy includes the bottom of the conduction band, then parabolic approximation of band diagram can be used, then the mode density $M(E)$ increases with energy. In the valence band, information related to the subbands are more difficult, because the coupled multiple bands that are increasing and difficult dispersion relations are needed.

By taking the derivatives wave vector $k$ over the energy $E(d k / d E)$ of (8), the number of the mode $M(E)$ is written as

$$
M(E)=\frac{\Delta E}{\Delta k \cdot L}=\frac{3 a t}{2 L}\left(\frac{4 E}{3 a t}-2 \beta^{2}\right)^{1 / 2},
$$

where $L$ is the length of the nanoribbon. Now taking into consideration of spin degeneracy, the number of conducting channels can be finalized as

$$
M(E)=2 \frac{\Delta E}{\Delta k \cdot L}=\frac{3 a t}{L}\left(\frac{4 E}{9 a t}-2 \beta^{2}\right)^{1 / 2} .
$$

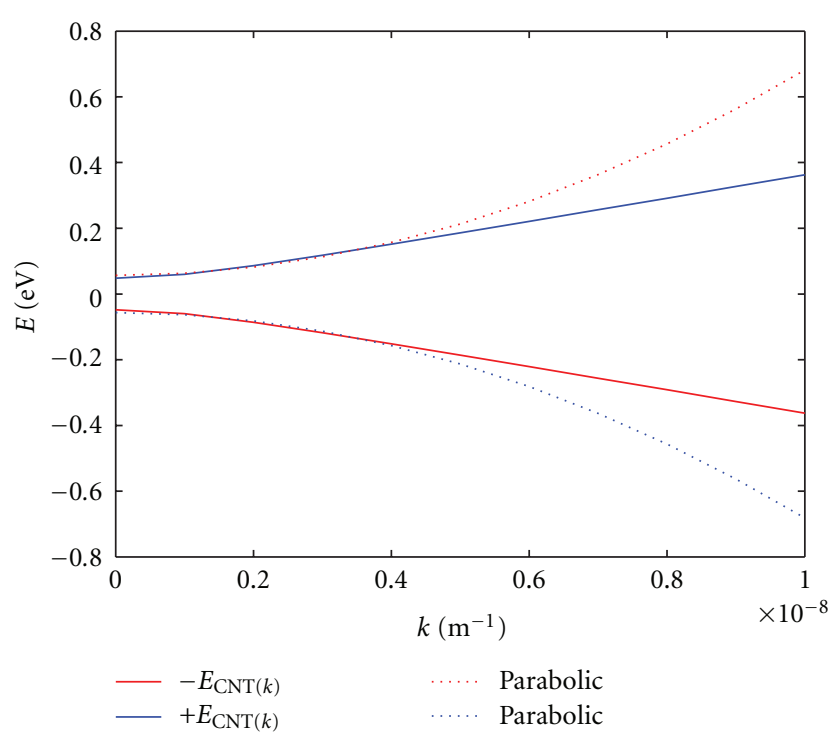

FIGURE 2: The band structure of GNR near the minimum energy is parabolic.

A region of lowest $G$ with respect to gate voltage in bulk graphene is calculated and matches to the minimum conductivity at the charge neutrality point, $V_{g}=V_{\text {Dirac }}$. That is a fundamental constant proportional to the Planck's constant and electron charge

$$
G_{0}=\frac{2 q^{2}}{h}
$$

where $q$ is electron charge and $h$ is Plank constant. In fact, levels of up spin and down spin in the small channels naturally with same energy as a degenerate level result in the minimum conductance two times larger than this amount which is equal to $2 G_{0}$. In the bad contact, conductance measured is always lower than this value. Based on the Landauer formula, the conductance on large channel can follow the ohmic scaling law but in the smaller size needs to apply two possible corrections on this law, firstly, interface resistance which is independent of the length. Secondly, conductance related to the width nonlinearly which depends on the number of the modes in the conductor that is quantized parameter, in Landauer formula, both of these features are corporate and conductance is

$$
G=\frac{2 q^{2}}{h} \int_{-\infty}^{+\infty} d E M(E) T(E)\left(-\frac{d f}{d E}\right)
$$

where $T$ is average probability of injected electron at one end will transmit to the other end, in our ballistic channel, this parameter is equal to one [20]. Replacement by the number of subbands (mode numbers) corporate with Fermi-Dirac distribution function conductance is related to the length of nanoribon as well

$$
G=\frac{3 q^{2}}{h} \frac{a t}{L}(3 a t)^{1 / 2} \int_{-\infty}^{+\infty}\left(E-\frac{2 \beta^{2}}{3 a t}\right)^{1 / 2} d\left(-\frac{1}{1+e^{\left(E-E_{F}\right) / k_{B} T}}\right) .
$$




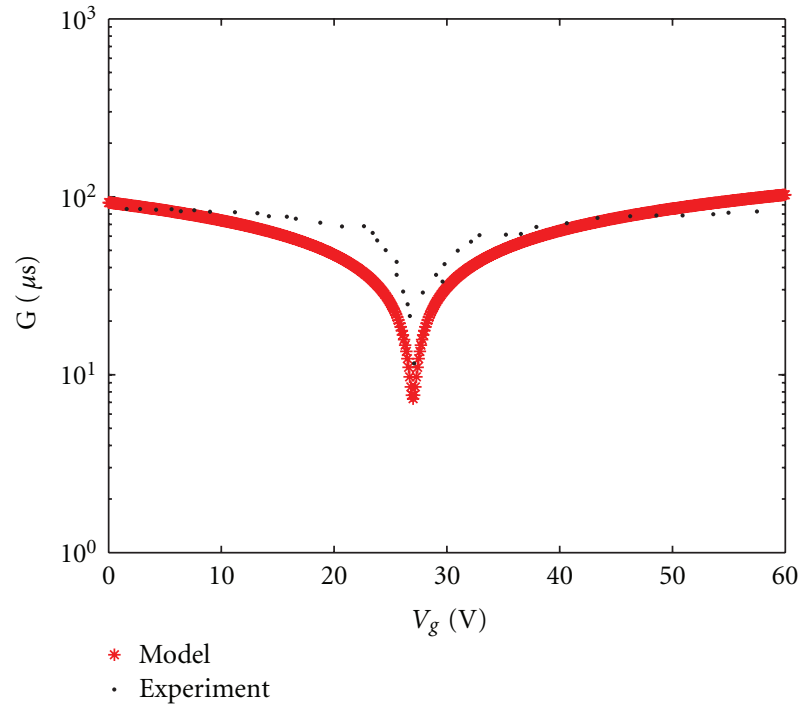

FIGURE 3: Comparison between GNR conductance model (Solid line) and experimental data (dotted line) displays good agreement between theoretical model and experimental data.

Temperature effect on nanoribbon conductance can be seen by changing the boundary of integral as follow

$$
\begin{aligned}
G= & \frac{3 q^{2}\left(3 \pi a^{3} t^{3} k_{B} T\right)^{1 / 2}}{h L} \\
& \times\left[\int_{0}^{+\infty} \frac{x^{-1 / 2}}{\left(1 /\left(1+e^{x-\eta}\right)\right)} d x+\int_{0}^{+\infty} \frac{x^{-1 / 2}}{\left(1 /\left(1+e^{x+\eta}\right)\right)} d x\right],
\end{aligned}
$$

where $x=\left(E-E_{g}\right) / k_{B} T$ and normalized Fermi energy is $\eta=\left(E_{F}-E_{g}\right) / k_{B} T$. Presenting Fermi-Dirac integral form of conductance is useful to understand the role of degenerate and nondegenerate regimes.

$$
G=\frac{3 q^{2}\left(3 \pi a^{3} t^{3} k_{B} T\right)^{1 / 2}}{h L}\left[\mathfrak{I}_{-1 / 2}(\eta)+\mathfrak{I}_{-1 / 2}(-\eta)\right] .
$$

GNR conductance model presented on (14) indicates minimum conductance near the charge neutrality point which is in good agreements with experimental results as shown in Figure 3. The presented model here provides possibility towards emerging grapheme-based quantum devices.

Low conductance with respect to the gate voltage indicates minimum conductivity at the charge neutrality point (known as Dirac point), in the neighborhood of the chargeneutrality, point resistance is higher and carriers follow linear energy-momentum dispersion relations.

Unlike the three-dimensional graphene, GNRs show a decrease in minimum conductance more than an order of magnitude at low temperatures as shown in Figure 4. Also the defeat of $G$ near the Dirac point recommends the energy gap in the GNR. However, significant shift of the minimum conductance at Dirac point may be effect on reduction of carrier mobility in graphene. The temperaturedependent conductance in GNRs, compare to that of the

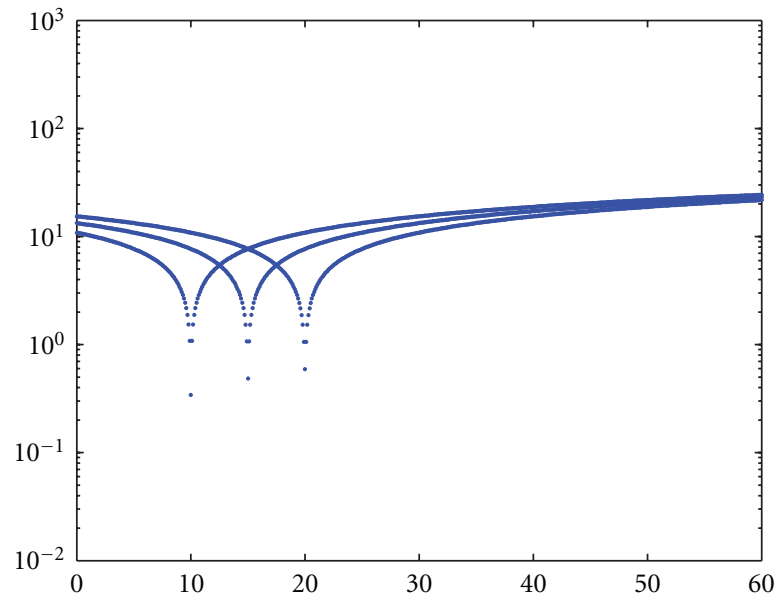

FIgURE 4: Conductance of GNRs as a function of gate voltage is plotted at different temperatures.

three-dimensional graphene samples, where conductance at Dirac point changes less with variation of temperature from $0 \mathrm{~K}$ to $300 \mathrm{~K}$ shows different performance [3].

Nondegenerate approximation on Fermi-Dirac integral can be used when Fermi level in band gap is far from conduction and valence band age more than $3 k_{B} T$. If the Fermi level lies inside the valance or conduction band or located $3 k_{B} T$ in the interior of the band edge, degenerate approximation has to be used. Moreover, GNR length effect can be discussed in terms of degeneracy phenomena, by increasing the length of GNR, nondegenerate approximation will be dominant similar to the conventional long channel device. In the nondegenerate limit, Fermi-Dirac integral can be converted into the exponential equation as

$$
G=\frac{3 q^{2}\left(3 \pi a^{3} t^{3} k_{B} T\right)^{1 / 2}}{h L}\left[e^{(\eta)}+e^{(-\eta)}\right] .
$$

Reduction of nondegenerate mobility near the Dirac point can be seen by dotted line in Figure 5, also it is shown as a gap region appears for $25<V g<30 \mathrm{~V}$. Near the Dirac point but outside of the gap region, the conductance balances with the width of the GNR. In the other word, narrower GNRs demonstrate the greatest dominance of minimum conductance at Dirac point [3].

As a device channel, the active GNR width contributes in charge transport, a narrower GNR with possible larger band gap makes them as a semiconducting device component [21]. However, in order to apply the GNR remarkable electrical appearance in nanoelectronic production, a band gap is highly required in graphene, therefore, strong demand is being made to explore the electrical properties of onedimensional graphene nanostructures. The Fermi level variation and number of conduction modes that contribute to the transport in GNRs also are governed by bias voltage between the source and drain contacts [3-6]. 


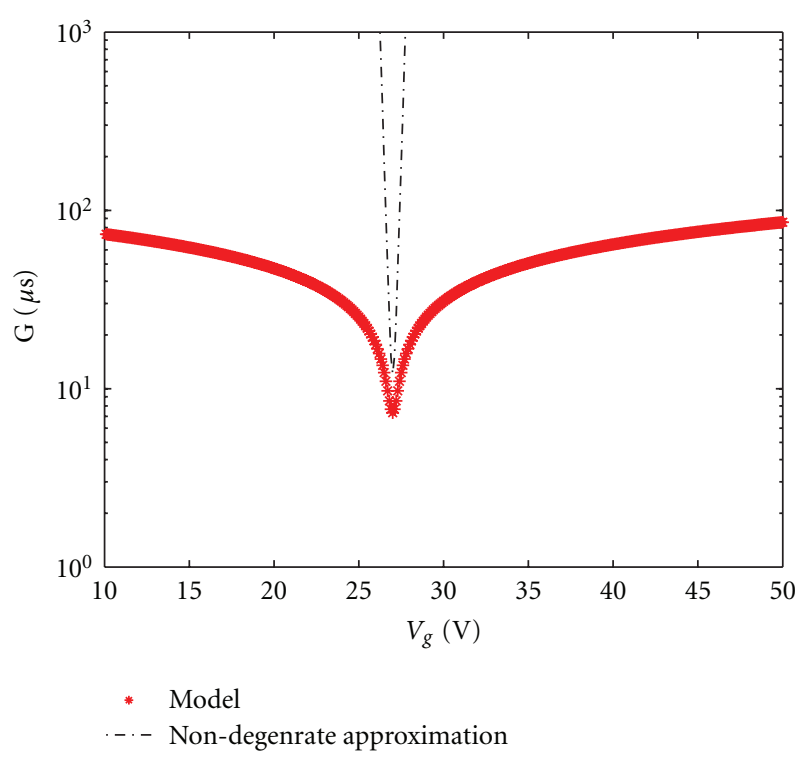

FIGURE 5: Nondegenerate approximations (dotted black line) near the Dirac point on comparison with real conductance (red line).

\section{Conclusion}

Based on quantum confinement effect granting parabolic band structure in semiconductor GNRs, conductance is a function of Fermi-Dirac integral which is based on Maxwell approximation in nondegenerate limit. GNR conductance at Dirac point illustrates minimum conductance at charge neutrality point which depends on temperature. Based on the presented model, GNR minimum conductance decreases by decreasing the temperature. Reduction of nondegenerate mobility near the charge neutrality point can be related to the minimum conductance near the Dirac point, however, outside of the Dirac point, the conductance balances with the width of the GNR.

\section{Acknowledgments}

The work is supported by postdoctoral fellowship scheme for the project "Nanoscale device modeling and simulation", faculty of Electrical Engineering, managed by the UTM Research Management Center (RMC). The authors would like to thank the MOSTI and RMC for cordially sponsoring this work.

\section{References}

[1] K. S. Novoselov, A. K. Geim, S. V. Morozov, et al., "Electric field in atomically thin carbon films," Science, vol. 306, no. 5696, pp. 666-669, 2004.

[2] K. S. Novoselov, A. K. Geim, S. V. Morozov et al., "Twodimensional gas of massless Dirac fermions in graphene," Nature, vol. 438, no. 7065, pp. 197-200, 2005.

[3] M. Y. Han, B. Özyilmaz, Y. Zhang, and P. Kim, "Energy bandgap engineering of graphene nanoribbons," Physical Review Letters, vol. 98, no. 20, Article ID 206805, 4 pages, 2007.
[4] Z. Chen, Y.-M. Lin, M. J. Rooks, and P. Avouris, "Graphene nano-ribbon electronics," Physica E, vol. 40, no. 2, pp. 228232, 2007.

[5] C. Stampfer, J. Güttinger, F. Molitor, D. Graf, T. Ihn, and K. Ensslin, "Tunable Coulomb blockade in nanostructured graphene," Applied Physics Letters, vol. 92, no. 1, Article ID 012102, 3 pages, 2008.

[6] F. Sols, F. Guinea, and A. H. C. Neto, "Coulomb blockade in graphene nanoribbons," Physical Review Letters, vol. 99, no. 16, Article ID 166803, 4 pages, 2007.

[7] C. Berger, Z. Song, T. Li et al., "Ultrathin epitaxial graphite: 2D electron gas properties and a route toward graphene-based nanoelectronics," Journal of Physical Chemistry B, vol. 108, no. 52, pp. 19912-19916, 2004.

[8] C. Berger, Z. Song, X. Li et al., "Electronic confinement and coherence in patterned epitaxial graphene," Science, vol. 312, no. 5777, pp. 1191-1196, 2006.

[9] B. Obradovic, R. Kotlyar, F. Heinz et al., "Analysis of graphene nanoribbons as a channel material for field-effect transistors," Applied Physics Letters, vol. 88, no. 14, Article ID 142102, 3 pages, 2006.

[10] K. Nakada, M. Fujita, G. Dresselhaus, and M. S. Dresselhaus, "Edge state in graphene ribbons: nanometer size effect and edge shape dependence," Physical Review B, vol. 54, no. 24, pp. 17954-17961, 1996.

[11] T. Ohta, A. Bostwick, T. Seyller, K. Horn, and E. Rotenberg, "Controlling the electronic structure of bilayer graphene," Science, vol. 313, no. 5789, pp. 951-954, 2006.

[12] R. Van Noorden, "Moving towards a graphene world," Nature, vol. 442, no. 7100, pp. 228-229, 2006.

[13] N. M. R. Peres, A. H. C. Neto, and F. Guinea, "Erratum: conductance quantization in mesoscopic graphene," Physical Review B, vol. 73, no. 23, Article ID 239902, 1 pages, 2006.

[14] D. Gunlycke, D. A. Areshkin, and C. T. White, "Semiconducting graphene nanostrips with edge disorder," Applied Physics Letters, vol. 90, no. 14, Article ID 142104, 3 pages, 2007.

[15] J. Fernández-Rossier, J. J. Palacios, and L. Brey, "Electronic structure of gated graphene and graphene ribbons," Physical Review B, vol. 75, no. 20, Article ID 205441, 8 pages, 2007.

[16] M. Lundstrom and J. Guo, Nanoscale Transistors, Springer, New York, NY, USA, 2006.

[17] S. Thornhill, N. Wu, Z. F. Wang, Q. W. Shi, and J. Chen, "Graphene nanoribbon field-effect transistors," in Proceedings of the IEEE International Symposium on Circuits and Systems (ISCAS '08), pp. 169-172, Seattle, Wash, USA, May 2008.

[18] M. T. Ahmadi, A. H. Fallahpour, V. Kouhdaragh, K. Dagahi, and R. Ismail, "Band structure effect on Carbon Nanotube Fermi energy," International Review of PHYSICS, vol. 3, no. 44, pp. 224-227, 2009.

[19] M. T. Ahmadi, R. Ismail, M. L. P. Tan, and V. K. Arora, "The ultimate ballistic drift velocity in carbon nanotubes," Journal of Nanomaterials, vol. 2008, no. 1, Article ID 769250, 8 pages, 2008.

[20] S. Datta, Electronic Transport in Mesoscopic Systems, Cambridge University Press, Cambridge, UK, 2002.

[21] A. Naeemi and J. D. Meindl, "Conductance modeling for graphene nanoribbon (GNR) interconnects," IEEE Electron Device Letters, vol. 28, no. 5, pp. 428-431, 2007. 

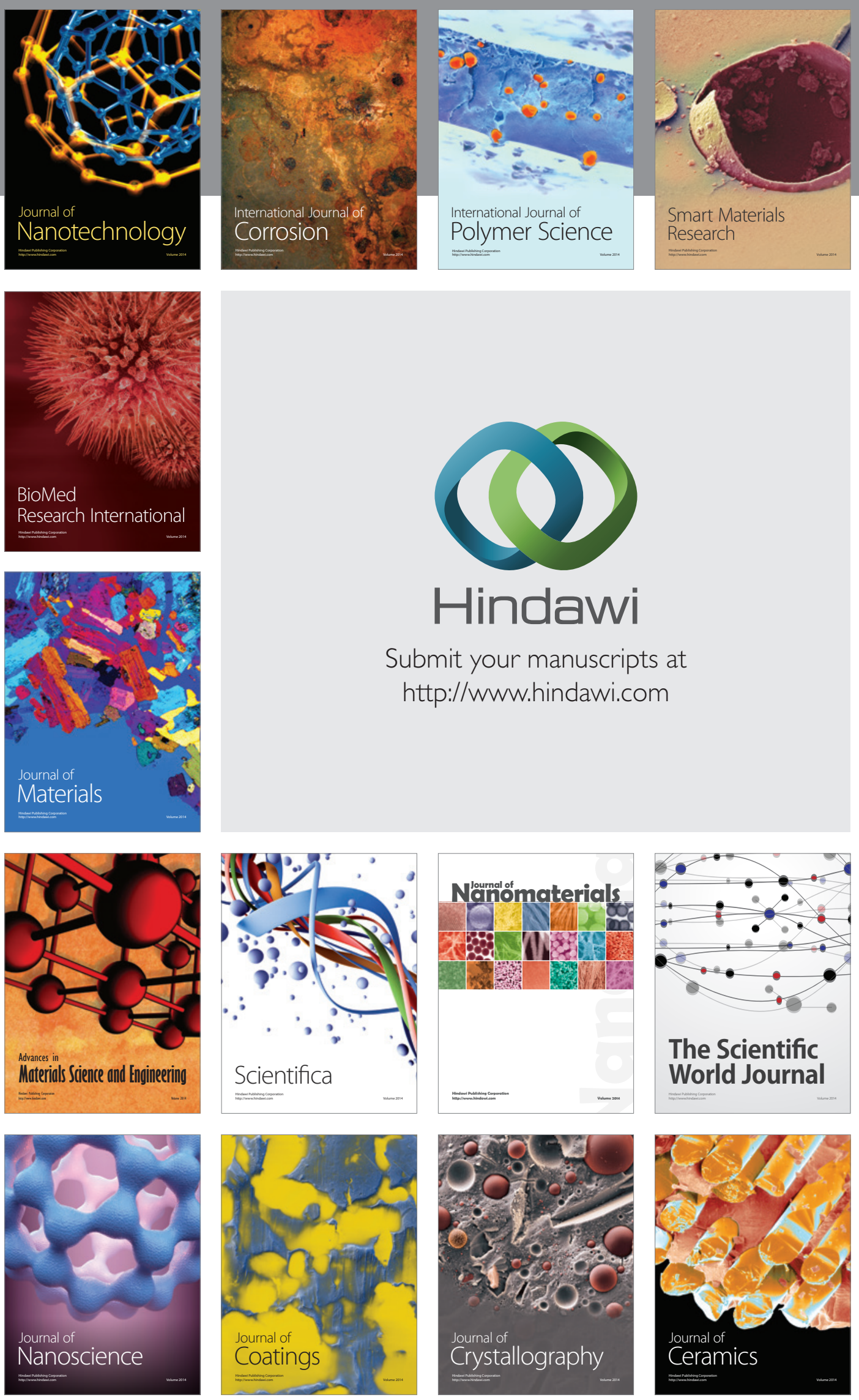

The Scientific World Journal

Submit your manuscripts at

http://www.hindawi.com

\section{World Journal}

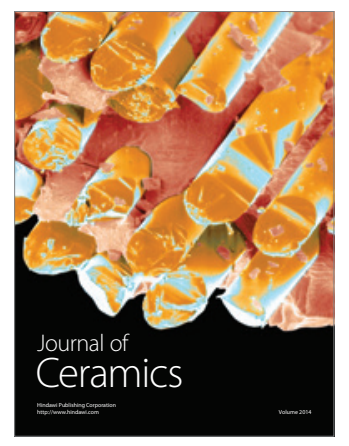

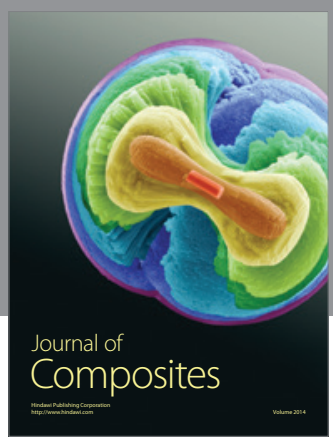
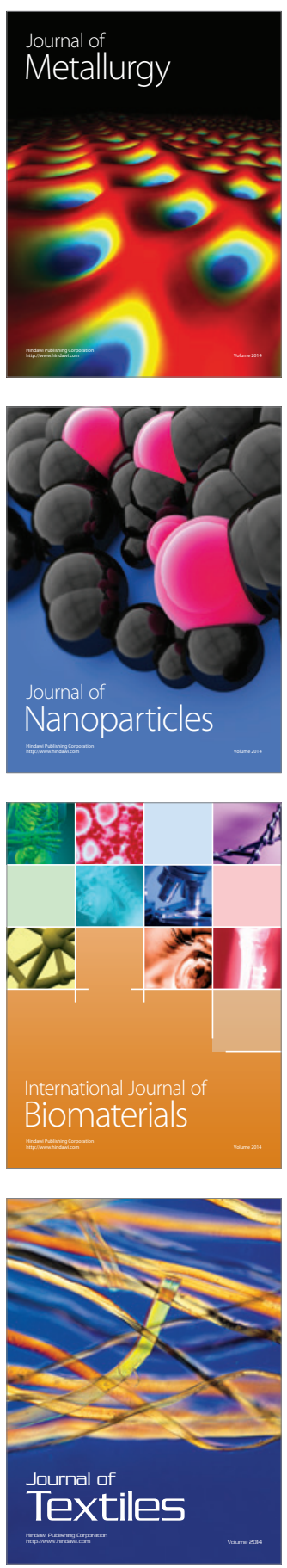